\title{
Evaluación de la exposición a ruido social en jóvenes chilenos
}

\author{
Assessment of social noise exposure in Chilean youth
}

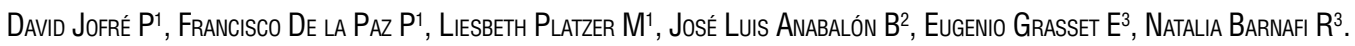

\section{RESUMEN}

Introducción: La exposición al ruido derivado de actividades de ocio y su repercusión sobre la audición humana, especialmente en jóvenes y en adolescentes, es un problema al cual la sociedad presta cada vez mayor atención. Debido a lo anterior realizamos un estudio para comparar el nivel de ruido al cual está expuesto un grupo de jóvenes chilenos respecto a estudios similares realizados en Europa.

Objetivos: Identificar actividades de entretención que puedan constituirse en actividades de riesgo para inducir una pérdida auditiva a largo plazo. Además, estudiar la exposición semanal derivada de las actividades de entretención más frecuentes en adolescentes de nuestro medio.

Material y método: Se realizó un estudio de prevalencia, encuestando a 243 jóvenes de Santiago. La exposición semanal al ruido se calculó en base al tiempo de exposición y el equivalente en $d B$ de intensidad de cada actividad. La exposición al ruido social registrada fue promediada a un total de 40 horas semanales. Por último se comparó el ruido semanal con el límite de riesgo laboral de 85 dBA.

Resultados: Se identificaron actividades de riesgo tales como ir a discotecas o conciertos de rock, tocar en una banda de rock y asistir a bares. Los resultados de la encuesta mostraron $30 \%$ de adolescentes chilenos expuestos a niveles de ruido superiores al límite considerado como de riesgo por exposición semanal.

Conclusiones: Al comparar los resultados de nuestro estudio con aquellos realizados previamente, se observa que en nuestros adolescentes existe un menor riesgo de daño por exposición a ruido respecto a jóvenes españoles y finlandeses. Es preciso estudiar aún más este fenómeno y tratar de establecer relaciones causales con medidas objetivas, permitiendo así prevenir y educar.

Palabras clave: Ruido, adolescentes, ruido social.

\begin{abstract}
Introduction: Exposure to leisure activities-related noise and its effects on human hearing, e especially in young adults and adolescents, represents a problem to which society is more and more sensitive. For this reason, we carried out a study in which we compared the noise level to which a group of young Chileans was exposed, with similar studies from Europe.
\end{abstract}

1 Médico Servicio de Otorrinolaringología, Pontificia Universidad Católica de Chile.

2 Tecnólogo Médico en Otorrinolaringología, Pontificia Universidad Católica de Chile.

3 Alumno Escuela de Medicina de la Universidad de Chile. 
Aim: The purpose of this study was to identify leisure activities that could pose a risk of inducing hearing loss in the long term. A second aim was to study the weekly noise exposure levels derived from the most frequent teenager leisure activities in our society.

Material and Method: A prevalence study was carried out by surveying 243 young individuals from Santiago. Weekly noise exposure level was calculated considering both the $d B$ intensity of each activity and the time exposed. Average social noise exposure registered was 40 hours per week. Intensity of the noise level per week was compared with the work risk threshold of $85 \mathrm{~dB}$.

Results: Risk activities such as attending rock concerts, discotheques or pubs were identified. The survey results show a 30\% of Chilean adolescents exposed to noise levels above the threshold considered a risk when exposed to it weekly.

Conclusions: A comparison of our results with those from the literature shows that the risk of damage due to noise exposure is smaller in our young population than in Spanish or Finnish teenagers. Further studies are needed to establish causal relationships with objective measures, allowing for education and prevention.

Key words: Noise, adolescents, social noise.

\section{INTRODUCCIÓN}

El término 'ruido' corresponde a un sonido inarticulado, por lo general desagradable ${ }^{1}$. En Otorrinolaringología se ha aplicado a cualquier sonido de un volumen suficientemente alto para tener potencial de daño en la audición².

La norma chilena establece como período máximo de exposición laboral a 8 horas a $85 \mathrm{~dB}$ $(A), 4$ horas a $90 \mathrm{~dB}(\mathrm{~A}) 02$ horas a $95 \mathrm{~dB}(\mathrm{~A})^{3,4}$.

Existen diversas fuentes de exposición a ruido no ocupacional o recreativo, siendo la música a niveles sonoros altos la más común para la mayoría de los jóvenes, escuchada ya sea en el hogar, fuera de él 0 a través de equipos musicales personales ${ }^{5}$.

Hay también otras fuentes de ruido como conducir motocicletas, tiro al blanco, uso de herramientas, etc., a las que están expuestos grupos menores de ellos. ${ }^{6}$

La exposición al ruido derivado de actividades de entretención ('ruido social') y su repercusión sobre la audición humana, especialmente en jóvenes, constituye un problema.

Sin embargo, el interés de los estudios científicos sobre el ruido y el daño que éste ejerce sobre la salud se ha centrado al ámbito laboral, siendo escasos los estudios sobre ruido social que es posible encontrar en la actualidad ${ }^{2,5-8}$.
Jokitulppo (1997) realizó una investigación en un grupo de jóvenes finlandeses entre 12 y 17 años. Los datos fueron analizados a partir de una encuesta que contenía preguntas acerca de las actividades de entretención realizadas por los participantes, el tiempo semanal dedicado a cada actividad y el nivel de ruido que representaba cada una?

El año 2001 Zenker y Barajas repitieron el estudio en España en jóvenes entre 14 y 24 años ocupando la misma encuesta ${ }^{6}$, encontrando que $65 \%$ de la población de jóvenes españoles estudiada tenía exposición a ruido dentro de rangos potencialmente peligrosos en comparación con $50 \%$ de la población de jóvenes finlandeses investigado por Jokitulppo. En la Figura 1 se observa una comparación de la distribución de la exposición semanal al ruido en españoles y finlandeses. Ésta fue calculada a partir del total de la muestra en relación a todas las actividades estudiadas, el tiempo dedicado a cada una de ellas, e intensidad para cada actividad.

\section{OBJETIVOS}

Nuestros objetivos generales fueron:

1. Identificar las actividades de entretención que puedan constituirse en actividades riesgosas de inducir una pérdida auditiva a largo plazo. 


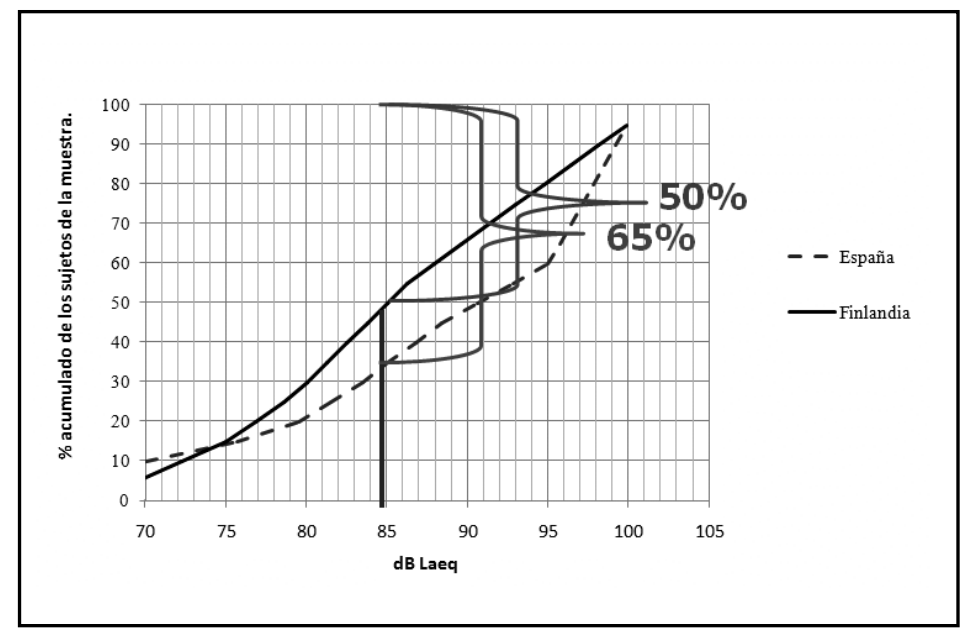

Figura 1. Distribución de la exposición semanal al ruido en jóvenes españoles y finlandeses.

2. Estudiar la exposición semanal derivada de las actividades de entretención más frecuentes en adolescentes de nuestro medio.

Nuestros objetivos específicos fueron:

1. Analizar los resultados de la encuesta aplicada a jóvenes chilenos.

2. Comparar nuestros resultados con los trabajos finlandeses y españoles.

\section{MATERIAL Y MÉTODO}

Se realizó un estudio de prevalencia. La recolección de datos se llevó a cabo, mediante la aplicación de una encuesta validada en Europa ${ }^{6,7}$, en jóvenes voluntarios de un colegio privado y estu- diantes universitarios de la ciudad de Santiago, con un tamaño de la muestra de 243 jóvenes.

La encuesta está diseñada presentando una tabla con varias entradas para que el sujeto evalúe: (Figura 2)

Su participación en determinadas actividades de entretención.

El tiempo dedicado a cada una de ellas.

La estimación subjetiva de la intensidad de cada actividad?

Con el fin de poder obtener valores objetivos de la intensidad de cada actividad se recurrió a la literatura disponible sobre este tema ${ }^{6,7}$.

La exposición semanal al ruido se calculó usando el tiempo de exposición y el equivalente en $\mathrm{dB}$ de intensidad de cada actividad (Figura 3).

\begin{tabular}{|c|c|c|c|c|c|c|c|}
\hline Actividad & $\begin{array}{c}\text { Dias de la } \\
\text { semana }\end{array}$ & $\begin{array}{c}\text { Horas } \\
\text { al dia. }\end{array}$ & $\begin{array}{c}1 \\
\text { Muy } \\
\text { silencioso }\end{array}$ & 2 & 3 & 4 & $\begin{array}{c}5 \\
\text { Muy } \\
\text { Ruidoso. }\end{array}$ \\
\hline Tocaren un grupo de música & & & & & & & \\
\hline Tocarun instrumento de música & & & & & & & \\
\hline Mezclar música (DJ, PC) & & & & & & & \\
\hline Escuchar MP3, walkman, etc. & & & & & & & \\
\hline EscucharTV & & & & & & & \\
\hline Andaren Moto & & & & & & & \\
\hline Deporte en gimnasio cerrado & & & & & & & \\
\hline Deporte al aire libre & & & & & & & \\
\hline Conciertos de Rock & & & & & & & \\
\hline Discotecas & & & & & & & \\
\hline Bares & & & & & & & \\
\hline Uso de herramientas & & & & & & & \\
\hline Tiro (caceria o deporte) & & & & & & & \\
\hline
\end{tabular}

Figura 2. Encuesta efectuada a jóvenes. 
La exposición al ruido social calculada finalmente fue promediada a un total de 40 horas semanales.

Por último se comparó el ruido semanal con el límite de riesgo laboral de $85 \mathrm{dBA}$.

\section{RESULTADOS}

Se realizaron 243 encuestas en jóvenes de la ciudad de Santiago, con una distribución por sexo de $45 \%$ masculino y $55 \%$ femenino (Figura 4 ). La edad promedio fue de 17 años 6 meses (rango: 15 y 26 años). En cuanto a la procedencia, 79\% (n: 191) provenía de colegios privados y el resto eran universitarios.

Se estudió el tiempo dedicado a las distintas actividades observando que las mayores cantidades de horas se ocupaban en: ver televisión, escuchar MP3 y mezclar (0 bajar de Internet) música. Los tiempos promedios expresados en horas semanales no aluden al total de los 243 jóvenes encuestados sino únicamente a quienes respondieron afirmativamente cada una de las actividades incluidas en la encuesta (Figura 5).

El total de los sujetos estudiados practicaba una 0 varias actividades ruidosas a lo largo de la semana. En general, el total de la muestra consideró a las discotecas y los conciertos de rock como las dos actividades más ruidosas. Otras actividades consideradas muy ruidosas fueron asistir a bares, y ser miembro de un grupo de música. La actividad menos ruidosa fue ver televisión y la práctica de algún deporte al aire libre (Figura 6).

En la Figura 7 se muestra la distribución estimada de exposición semanal para la suma de todas las actividades de ocio analizadas en este estudio.

\begin{tabular}{|c|c|c|}
\hline Actividad & $\begin{array}{c}\text { Intervalo de } \\
\text { intensidad. } \\
\text { (1 a 5) }\end{array}$ & Valor máximo de Laeq \\
\hline Tocar en un grupo de música & $90-135$ & 105 \\
\hline Tocarun instrumento de música & $75-114$ & 105 \\
\hline Mezclar música (DJ, PC) & $73-110$ & 100 \\
\hline Escuchar MP3, walkman, etc. & $85-120$ & 100 \\
\hline EscucharTV & $70-100$ & 100 \\
\hline Andar en Moto & $70-112$ & 105 \\
\hline Deporte en gimnasio cerrado & $70-112$ & 100 \\
\hline Deporte al aire libre & $70-112$ & 100 \\
\hline Conciertos de Rock & $84-125$ & 105 \\
\hline Discotecas & $84-125$ & 105 \\
\hline Bares & $84-125$ & 105 \\
\hline Uso de herramientas & $60-115$ & 105 \\
\hline Tiro (cacería o deporte) & 135 & 105 \\
\hline
\end{tabular}

Figura 3. Niveles sonoros equivalentes para las actividades estudiadas.

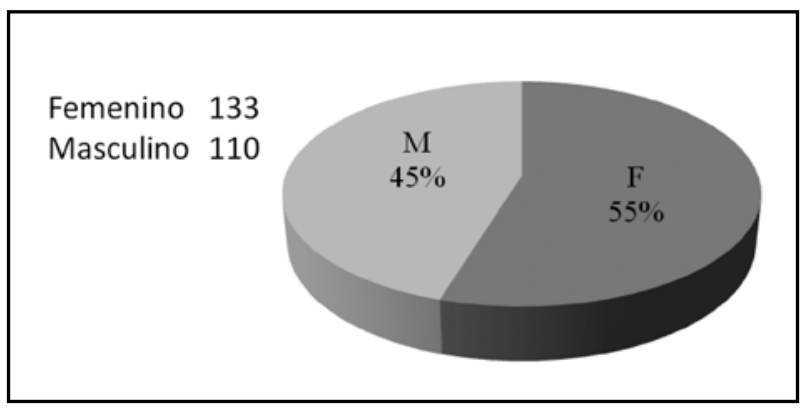

Figura 4. Distribución por género de la muestra. 


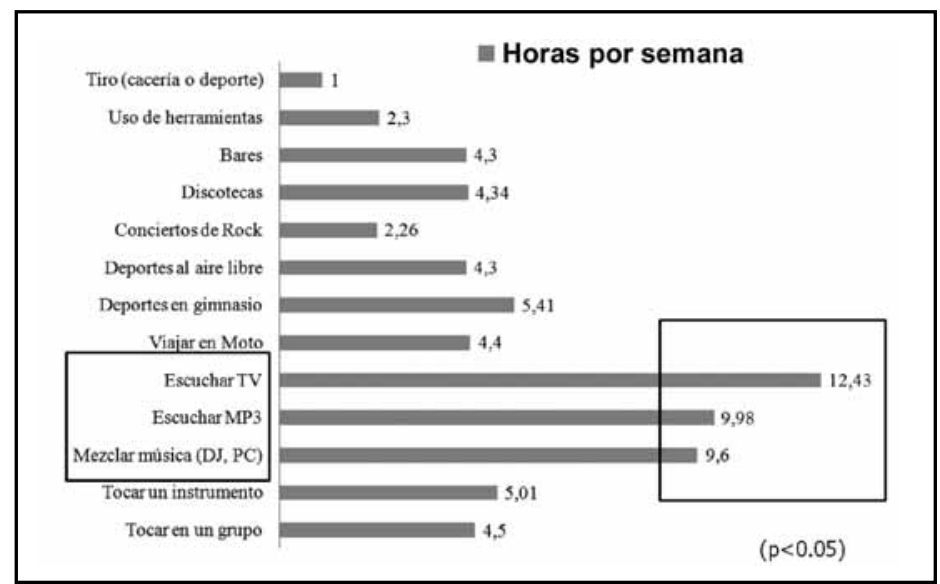

Figura 5. Tiempo dedicado a cada una de las diferentes actividades analizadas en este estudio.

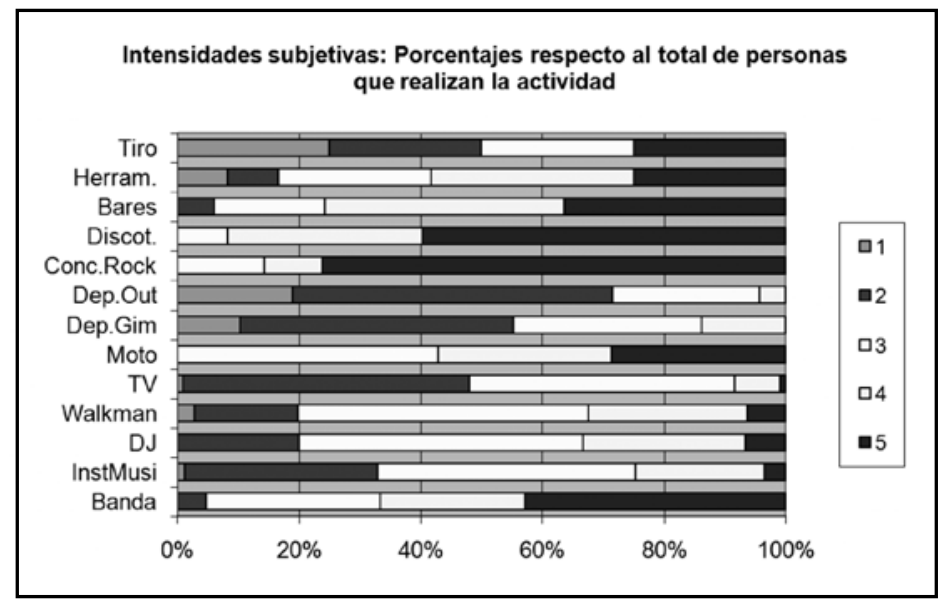

Figura 6. Actividades estudiadas expresadas en una escala de 1 (muy silenciosa) a 5 (muy ruidosa).

Ésta fue calculada a partir del total de la muestra en relación a todas las actividades estudiadas, tiempo dedicado a cada una de ellas, e intensidad para cada actividad. La media fue de 85,1 dB A. No se encontraron diferencias significativas entre ambos sexos. El límite de riesgo de 85 dB A (ISO 1999) fue alcanzado por el $30 \%$ de los sujetos de este estudio.

\section{DISCUSIÓN Y CONCLUSIONES}

Este trabajo muestra como un gran porcentaje de los jóvenes participantes (30\%) está expuesto a intensidades sonoras derivadas de actividades de entretención superiores a las consideradas como de riesgo laboral (exposición a 85 dBA durante 40 horas semanales).

Comparando nuestra investigación con trabajos previos, los adolescentes del presente estudio tienen menos riesgo que jóvenes españoles y finlandeses.

Se identifican actividades de riesgo: ir a discotecas 0 conciertos de rock, tocar en una banda de rock y asistir a bares. Es hacia estos grupos de riesgo donde es factible orientar políticas de prevención.

Se debe estudiar en profundidad este fenómeno y tratar de establecer relaciones causales con medidas objetivas y, así, prevenir y educar.

Los desafíos futuros que nos planteamos son:

- Realizar este estudio en una muestra representativa de la población nacional. 


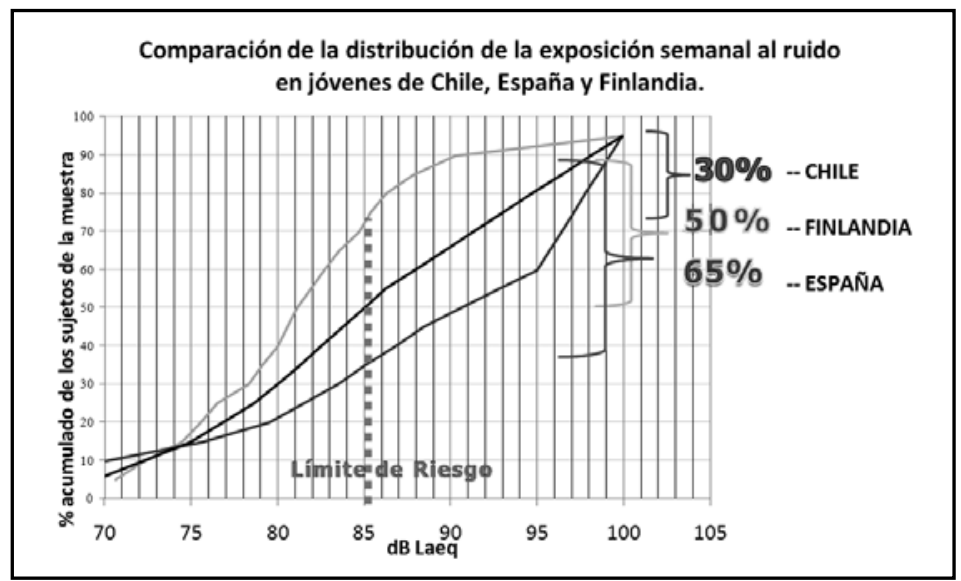

Figura 7. Distribución comparativa de la exposición semanal a ruido.

- Validar este instrumento de medición (encuesta) para que sea totalmente adecuado a la realidad nacional.

- Seguir, en lo posible, a un grupo a largo plazo (estudio de cohorte).

\section{BIBLIOGRAFÍA}

1. Diccionario de la Lengua Española. Vigésima segunda edición, España, 2001.

2. Ising H, Babisch W, Hanee J, Kruppa B. Loud music and hearing risk. Journal of Audiological Medicine 1997; 6: 123-33.

3. Reglamento sobre Condiciones Sanitarias y Ambientales Básicas en los Lugares de Trabajo. CONAMA. Decreto Supremo No 594/ 1999.
4. ISO 1999. Acoustic determination of occupational noise exposure and estimation of noiseinduced hearing impairment. Geneve: ISO, 1990

5. Dalton DS, Cruickshanks KJ, Wiley TL, Klein KE, KLEIN R, TweEd TS. Association of leisure-time noise exposure and hearing loss. Audiology 2001; 40: 1-9.

6. ZenkeR et AL. La exposición a ruido por actividades de ocio en adolescentes. Rev Log Fon Audiol 2001; xxi(4): 173-80.

7. Jokitulppo JS, BJörk EA, AKaAn-Penttilä E. Estimated leisure noise exposure and hearing symptoms in finnish teenagers. Scand Audiol 1997; 26: 257-62.

8. Davis AC, Lovell EA, Smith PA, Ferguson MA. The contribution of social noise to tinnitus in young people: A preliminary report. Noise \& Health 1998; 1: 40-6. 